\title{
Coherence: The Measurement and Application of Brain Connectivity
}

\author{
Scott L. Decker ${ }^{1 *}$, Paul T. Fillmore ${ }^{2}$, and Alycia M. Roberts ${ }^{3}$ \\ ${ }^{1}$ University of South Carolina, Department of Psychology, Columbia, South Carolina, USA \\ ${ }^{2}$ Baylor University, Department of Communication Sciences and Disorders, Waco, Texas, USA \\ ${ }^{3}$ Rainbow Babies \& Children's Hospital, Division of Developmental and Behavioral Pediatrics \& Psychology, Cleveland, \\ Ohio, USA
}

\begin{tabular}{|c|c|}
\hline \multicolumn{2}{|c|}{ Abstract } \\
\hline \multicolumn{2}{|c|}{$\begin{array}{l}\text { While much knowledge has been gained by the endeavor to link specific brain sites with specific cognitive } \\
\text { functions, modern conceptualizations of brain activity focus much more on the function of networks of brain } \\
\text { regions. A key construct in defining these networks has been the study of connectivity across regions. In this } \\
\text { review, we discuss several methods of measuring connectivity and focus primarily on the utility of } \\
\text { electroencephalographic (EEG) coherence. While over- and under-connectivity have been related to numerous } \\
\text { clinical phenomena, we focus our discussion on the role of connectivity in reading and language disorders, and } \\
\text { present a Neurocognitive Connectivity (NCC) framework for understanding these disorders. We argue that EEG } \\
\text { coherence presents a unique target for treatment of these and other populations, in that the ability to modulate } \\
\text { connectivity via EEG neurofeedback has been shown to be of significant clinical utility. }\end{array}$} \\
\hline \multicolumn{2}{|c|}{ Keywords: electroencephalography; coherence; neurocognitive connectivity; neurofeedback } \\
\hline \multicolumn{2}{|c|}{$\begin{array}{l}\text { Citation: Decker, S. L., Fillmore, P. T., \& Roberts, A. M. (2017). Coherence: The measurement and application of brain connectivity } \\
\text { NeuroRegulation, 4(1), 3-13. http://dx.doi.org/10.15540/nr.4.1.3 }\end{array}$} \\
\hline $\begin{array}{l}\text { *Address correspondence to: Scott L. Decker, University of South } \\
\text { Carolina, Department of Psychology, Hamilton College, Rm 350, } \\
1512 \text { Pendleton St., Columbia, SC 29208, USA. Email: } \\
\text { sdecker@mailbox.sc.edu }\end{array}$ & $\begin{array}{l}\text { Edited by: } \\
\text { Rex L. Cannon, PhD, Neurogifted, Boynton Beach, Florida, USA }\end{array}$ \\
\hline $\begin{array}{l}\text { Copyright: @ 2017. Decker et al. This is an Open Access article } \\
\text { distributed under the terms of the Creative Commons Attribution } \\
\text { License (CC-BY). }\end{array}$ & $\begin{array}{l}\text { Reviewed by: } \\
\text { Rex L. Cannon, PhD, Neurogifted, Boynton Beach, Florida, USA } \\
\text { Randall Lyle, PhD, Mount Mercy University, Cedar Rapids, lowa, } \\
\text { USA }\end{array}$ \\
\hline
\end{tabular}

\section{Introduction}

Linking cognitive and behavioral functions with specific regions of the brain through case studies of individuals with brain injury has been the primary basis of understanding "brain-behavior" relationships in neuropsychology. This dates back at least as far as Pierre Paul Broca (1861), who famously attributed specific deficits in the production of speech to corresponding damage to the inferior frontal lobe. Although neuropsychological studies such as Broca's have been valuable, technological advances in neuroimaging have drastically expanded the types of questions we can ask about cognition, especially in the healthy brain. For example, neuroimaging techniques have uncovered and refined theories about brain areas being "dedicated" to some domains of cognition, such as face processing (Kanwisher, McDermott, \& Chun, 1997), phonological decoding (Boukrina, Barrett, Alexander, Yao, \& Graves, 2015; He at al., 2013,), and the planning of motor speech (Dronkers, 1996; Richardson, Fillmore, Rorden, LaPointe, \& Fridriksson, 2012). However, on the whole, neuroimaging studies have also highlighted the limitations of simple localization perspectives of brain functions in demonstrating that most cognitive functions are not localized to just one area, but rather distributed across different regions of the brain.

Moving beyond simplistic theories of single-site localization, neuroimaging investigations of brain function have revealed that even relatively simple 
cognitive functions involve a complex and dynamic pattern of brain network activation across diverse regions of the brain. This complexity in patterns of brain activation parallels the complexity of human cognition found in even mundane everyday endeavors. Not only refuting simplistic localization theories of brain functioning, contemporary neuroimaging research also provides suggestion of deeper principles of brain function to explain cognition. Indeed, brain-behavior relationships extend beyond merely understanding the relationships between brain injury and behavior. Utilizing both structural and functional magnetic resonance imaging (MRI), numerous anatomical and functional properties of the brain have been uncovered. Most commonly, these studies involve combining detailed pictures of structure (e.g., T1weighted images), collected over several minutes, with estimates of blood flow over time (i.e., bloodoxygenation level-dependent [BOLD] contrasts), collected every several seconds. While this works well in many contexts, many cognitive functions unfold on timescales of tens or hundreds of milliseconds, requiring additional sources of information to fully understand them. Electromagnetic imaging techniques such as electroencephalography (EEG) and magnetoencephalography (MEG) fill this gap well, and have excellent temporal resolution (e.g., Breier , Simos, Zouridakis, \& Papanicolaou, 1999; Thierry, Boulanouar, Kherif, Ranjeva \& Démonet, 1999), thus providing unique perspectives on the function of brain "networks."

One important discovery in the past few decades has been the role of brain networks as an intermediary link between brain structure, cognition, and behavior. Understanding functions of the brain in terms of networks rather than specific anatomical structures has been a considerable development in modern neuroscience (Bullmore \& Sporns, 2009). There are numerous networks in the brain, without clear differentiation; however, the various networks are characterized by specific patterns of connectivity (Sporns, 2011; van den Heuvel \& Sporns, 2013). Similar to how different cities are connected by a network of airports, brain networks have hubs or central nodes with high connectivity, and other regions with low connectivity. The specific model of network connectivity in the brain has been described as a "small-world" network (Bassett \& Bullmore, 2006).

More importantly, connectivity, or lack thereof, in brain networks has proven to be an important theoretical construct with considerable applied applications. An emerging view in contemporary neuroscience is that many functional neurocognitive deficits for which individuals seek treatment are caused by problems in brain connectivity within specific brain networks, such as those that are important for academic learning (Paulesu et al., 1996; Rippon, Brock, Brown, \& Boucher, 2007), sustaining attention (Kucyi, Hove, Esterman, Hutchison, \& Valera, 2016), social communication (Coben, Clarke, Hudspeth, \& Barry, 2008; Grossmann, 2015), and fulfilling activities of daily living (Bieńkiewicz, Brandi, Goldenberg, Hughes, \& Hermsdörfer, 2014).

Despite the numerous studies demonstrating the importance of brain connectivity for different clinical conditions, measurement of brain connectivity has not typically been incorporated in general applications of psychological diagnostic procedures. The problem appears twofold. First, the rationale for including measures of brain network connectivity and how such measures can be connected to behavior is not clearly understood. Second, the measurement of brain connectivity is often confusing and also not well understood.

The rationale for differentially connected brain networks as an intermediary between brain structure and behavioral functioning is not derived from any one particular study but inducted through hundreds of studies (i.e., Bullmore \& Sporns, 2009; Fox et al., 2005; Mišić, \& Sporns, 2016, etc.). Collectively, broad assumptions are emerging that clarify the nature of the brain, brain-behavior relationships, and clinical applications thereof. While far from definitive, Table 1 provides an attempt to logically derive the role of connectivity through a list of assumptions, each supported by modern neuroscientific research.

\section{Table 1 \\ Assumptions regarding brain connectivity and cognitive functions.}

1. For any given cognitive function, there are multiple brain structures or sets of structures that are primarily involved in performing that function.

2. In most cases, these areas are functionally (and often structurally) connected, forming a specific network.

3. Dysfunction of a network via over- or underconnectivity will result in reduced proficiency in cognitive functions reliant upon connectivity of the involved brain regions. 
As derived from Table 1, there is an intricate relationship between "cognition" and "brain connectivity." In recognizing the emerging importance of the link between cognition and measures of brain connectivity, the term Neurocognitive Connectivity (NCC) will be used to provide a framework for linking cognition with brain connectivity through the assumptions of Table 1 . The NCC framework implicitly suggests that while having some theoretical importance when measured in isolation, brain connectivity is of primary interest when it can be linked to cognition or behavior. Similarly, therapeutic techniques for changing brain connectivity with no relevance for cognition or behavior are of little clinical value. Thus, the NCC framework is used to provide explicit assumptions regarding the clinical utility of measuring brain connectivity as a link to measures of cognition. Theoretically, NCC provides integration across empirical findings that extend beyond neuroscience and include development, cognition, genetics, and behavior. The remaining sections of this manuscript will provide additional details of the NCC framework and its clinical application. Additionally, we will discuss specific methods of objectively measuring and/or modulating brain network connectivity, focusing primarily on a metric referred to as EEG coherence (Bowyer, 2016).

\section{Importance of Connectivity}

Although the above rationale provides a strong theoretical foundation for an increased focus on the investigation of brain connectivity, the details of how we define and measure connectivity can be quite varied. Structural connectivity is often measured by MRI and diffusion tensor imaging (DTI), and yields information about both local and global directionality (via maps of fractional anisotropy; e.g., Feldman, Lee, Yeatman, \& Yeom, 2012; Lebel et al., 2013) as well as robustness of connections between defined points (via fiber-tracking methods; e.g., Vandermosten et al., 2012). While structure provides a vital substrate for the presence of networked brain function, it does not give the full picture of which networks actually exist, performing coordinated functions. Thus, the notion of functional connectivity has gained great traction in recent years (Glasser et al., 2016; Smith et al., 2013), including spawning large-scale projects to systematically map the human functional brain networks and their key nodes (http://www.humanconnectome.org/; Glasser et al., 2016). The term functional connectivity is used to describe the correlated neuronal activity of these various regions (Bowyer, 2016). However, this term is not only conceptual but also refers to the measurement basis of brain connectivity using fMRI and EEG methodologies. Functional connectivity refers to the cross-temporal correlation of measured brain activity in different regions of the brain (Bowyer, 2016; Honey et al., 2009). While much of the work in defining functional connectivity has utilized resting-state fMRI (RS-fMRI; e.g., Smith et al., 2013), as noted above, more temporally sensitive methods such as EEG and MEG are also necessary to evaluate how functional connectivity might change over brief timescales (Bowyer, 2016; also see discussion of functional versus effective connectivity in Friston, 2011). Coherence is one commonly used metric for deriving functional brain connectivity in EEG, which will be discussed in detail below.

\section{Defining Coherence}

Electroencephalographic measures provide excellent temporal resolution of brain activity and are based on electrical properties of the brain as measured by electrodes on the scalp. The measured electrical potentials on the scalp are small (microvolts: $u \mathrm{~V}$ ) and can be decomposed into frequency bands (i.e., Delta, Theta, Alpha, Beta, Gamma) or further into single hertz bins generally via the Fast Fourier Transform (FFT). The measured microvolts are typically squared $\left(u V^{2}\right)$ to derive Absolute Power, which is typically used as an underlying indicator of brain activation.

Coherence measures, on the other hand, quantify the degree of association between two brain regions, which is used to infer a functional relationship between two different regions of the brain. Similar to a correlation coefficient, coherence measures take values between 0 (no coherence) and 1 (absolute coherence). Coherence is calculated following transformation from the time domain to the frequency domain, and compares similarity of the power spectra, with regions showing greatest similarity being assumed to be the most functionally connected. It incorporates information on synchrony including both amplitude and phase, but is independent of power (Bowyer, 2016).

Most commonly, EEG coherence describes the interrelationship between two surface electrodes, though summaries such as site coherence (the average coherence for one electrode's coherences to all others) or global coherence (the average of all site coherences) can be useful; see Kaiser (2008) for a more comprehensive review. Recent methods (e.g., LORETA/eLORETA; Pascual-Marqui, Michel, \& Lehmann, 1994; Pascual-Marqui et al., 2011) also allow coherence to be estimated between brain 
regions themselves, using source analysis to infer the generators of EEG signals. In its most common formulation, EEG coherence is calculated by the form:

$$
y^{2} x y(f)=\frac{(G x y(f))^{2}}{(G x x(f) G y y(f))}
$$

where $G x y(f)$ is the cross-power spectral density and $\operatorname{Gxx}(\mathrm{f})$ and Gyy(f) are the respective auto-power spectral densities (Thatcher, Krause, \& Hrybyk, 1986). Though summarizing frequency content necessarily requires a defined time window, which is commonly at least tens of seconds for quantitative EEG (qEEG) analyses (Bowyer, 2016), these time windows can be shortened to allow for near-realtime estimates of coherence, making coherence a malleable metric for use in neurotherapeutic contexts such as neurofeedback.

Given the dynamic properties of brain activity, it would seem the correspondence between distantly located electrodes on different regions of the scalp would be, on the whole, erratic and unreliable. Surprisingly, coherence measures have been found to be quite reliable. For instance, reliability coefficients above $r=.80$ were first found in 1961 (Adey, Walter, \& Hendrix, as cited in Thatcher, 2010), with many later studies finding similar levels of stability (Cannon et al., 2012; Chabot et al., 1996; Corsi-Cabrera, Galindo-Vilchis, del-Río-Portilla, Arce, \& Ramos-Loyo, 2007; Corsi-Cabrera, SolísOrtiz, Guevara, 1997; John, 1977; Roberts, Fillmore, \& Decker, 2016; Thatcher, Walker, Biver, North, \& Curtin, 2003; though see Gudmundsson, Runarsson, Sigurdsson, Eiriksdottir, \& Johnsen, 2007, for an alternate perspective). One study (Fernández, Harmony, Rodríguez, Reyes, Marosi, \& Bernal, 1993) reported coherence reliability coefficients as high as $r=.95$ for both resting state and a verbal cognitive task, even with a test-retest interval of 1 month. Indeed, due to its high reliability, coherence is often targeted in neurofeedback treatment (i.e., Friedrich et al., 2014; Gruzelier, 2014; Keizer, Verment, \& Hommel, 2010). However, as demonstrated in Roberts, Fillmore, and Decker (2016), the effects of such a treatment protocol are highly dependent on the reliability of the targeted metric itself.

Several factors have been shown to affect coherence reliability. For instance, Shaw (1984) found that coherence was higher in eyes-closed than eyes-open resting state. This difference was the most pronounced in the alpha range; in which eyes-closed coherence reliability coefficients approached unity. Other patterns have emerged as well, including interhemispheric and gender differences in coherence (i.e., Gootjes, Bouma, Van Strien, Scheltens, \& Stam, 2006; Koles, Lind, \& FlorHenry, 2010; Miskovic, Schmidt, Boyle, \& Saigal, 2009; Tucker, Roth, \& Bair, 1986). Thatcher et al. (1986) also reported reliable patterns of coherence, which prompted the proposal of a twocompartmental model of coherence describing the importance of different types of cells for short- (i.e., basal dendrites) and long- (i.e., pyramidal cells) range communication.

\section{Neurocognitive Connectivity and Clinical Connections}

To demonstrate the basic assumptions of the importance of brain connectivity, a simplified demonstration involving reading cognition will be given and further expanded to discuss clinical implications for understanding Specific Learning Disabilities. The applicability of the framework for other neurodevelopmental disorders will also be discussed.

First, reading is a multi-dimensional cognitive task, and the specific cognitive demands change throughout its development. The early stages of reading involve "word decoding." Decoding involves, first, a visual analysis of letters and visual recognition of letter patterns or groups of letters. Next, letter groups must be associated with language sounds (phonology). To read a word, the letter sounds of different letters in the word must be blended. Finally, the blended letter sounds must be recognized as a word that is already stored in the individual's vocabulary (lexical semantics).

Specific cognitive processes involved in word decoding are linked to specific brain networks in different areas of the brain. First, the visual analysis of letters primarily involves brain networks beginning in the occipital lobe in the most posterior region of the brain. In contrast, the second step of phonology involves auditory sound representations that are primarily localized in the temporal regions of the brain, more specifically in the superior temporal region. Third, closely associated with auditory sound representations are the receptive languages areas, which involve networks in close proximity to auditory sound representation regions because language is learned through sound. However, language goes beyond sound to involve semantic representations or word meanings, which involve even more distributed networks in the brain. Thus, reading involves all the assumptions of an NCC 
framework as provided in Table 1 (see also Figure 1 for a pictorial representation of the NCC framework as it applies to reading). First, word decoding involves cognitive processes in different regions of the brain, which subsequently involve networks from different regions of the brain. Second, these different networks involved in reading must have functional connections for the normal development of reading to occur. Finally, reduced connectivity between brain networks involved in specific learning tasks reduces learning efficiency. Reduced learning efficiency of academic task(s) due to individual differences in atypical brain connectivity is the underlying cause of specific learning disabilities (SLD).

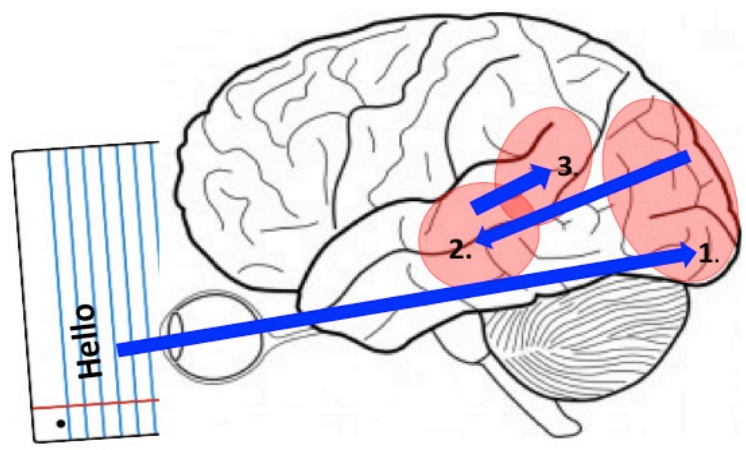

1. Decode: Visual analysis of letters and visual recognition of letter patterns occurs in the occipital lobe after the individual has viewed the word.

2. Phonology: Visual letter groups are associated with the language sounds and sound blending occurs primarily in the superior temporal lobe.

3. Semantics: Blended sounds are compared to words in stored vocabulary in neurological areas in close proximity to where phonological processing occurred.

Figure 1. NCC Framework of Word Recognition: Corresponding cognitive and coherence measures in different brain regions.

Numerous studies involving brain imaging support an NCC framework for SLD. One of the first studies investigating brain connectivity differences between children with dyslexia and typically developing peers found that children with dyslexia had "disconnected" language areas of the brain that corresponded to deficits in phonology (Paulesu et al., 1996). Here, it was proposed that weak connectivity between the anterior and posterior brain regions in the left hemisphere resulted in phonological deficits characteristic of many children with reading problems. Sally Shaywitz's work has also consistently demonstrated functional connectivity disruptions in the brains of individuals with dyslexia (e.g., Shaywitz, B. A., et al., 2002; Shaywitz, S. E., et al., 1998). Differences in brain connectivity in children with learning disabilities has also been linked to white matter structures of the brain, which serve as the major "highways" for connecting different brain regions (Silani et al., 2005; Temple, 2002). Additionally, reduced functional connectivity has been associated with deficits in integrating orthography and phonology in children with dyslexia (Cao, Bitan, \& Booth, 2008) and has been predictive of differences between children with dyslexia and neurotypical readers (Quaglino et al., 2008). Moreover, specific patterns of brain connectivity are linked to specific types of learning problems (Fields, 2008; Pugh et al., 2000).

Additionally, the NCC framework is not limited to reading disabilities. Recent research has demonstrated differentiated functional connectivity in brain regions involved in word processing amongst fMRI data for children with dysgraphia and oral and written language learning disabilities (Berninger, Richards, \& Abbott, 2015). Disruptions in neurocognitive connectivity have also been found in children with developmental dyscalculia (RosenbergLee et al., 2015). These networks often dissociate from those important for language-based SLD. For example, it has been found that individuals with math learning disabilities (MLD) exhibit disturbances in the left parietal and prefrontal brain areas (Geary, 2013). Another study found children with dyscalculia display decreased fractional anisotropy (a marker of white matter integrity) in the superior longitudinal fasciculus, as well as significant insufficiencies in fibers of the superior longitudinal fasciculus-a tract theorized to provide essential connections for numerical processing (Kucian et al., 2014). This is in contrast to children with dyslexia who typically have reduced connectivity in the left occipitotemporal cortex (Paulesu, Danelli, \& Berlingeri, 2014). Many other studies have also highlighted the ways in which brain areas implicated in dyscalculia are different than those in dyslexia, due to the different neurocognitive demands inherent in learning math and reading (Ashkenazi, Black, 
Abrams, Hoeft, \& Menon, 2013; Butterworth, Varma, \& Laurillard, 2011; Kucian \& von Aster, 2015). This demonstrates the flexibility in network characterization for SLD via the NCC framework.

The NCC perspective for viewing cognitive deficits as a result of disconnection of brain networks not only provides a fresh perspective for understanding SLD but also for grasping neurological disorders at large (Stam, 2014). Abnormal patterns of brain connectivity have been linked to numerous developmental and psychiatric conditions, and reduced symptomatology in these conditions is dependent on the normalization of brain network connectivity (Voytek \& Knight, 2015). For example, atypical patterns of connectivity have been found in individuals with schizophrenia (Su, Hsu, Lin \& Lin, 2015), epilepsy (Widjaja et al., 2015), and Alzheimer's Disease (Qin et al., 2015), to name a few. The NCC framework may help to devise new and better identification and/or treatment options for individuals with these (and other) neuropsychiatric disorders.

Given the relevance of the NCC framework in the understanding of neuropsychiatric disorders, it follows logically that metrics capable of characterizing and/or modulating brain connectivity would be ideally suited for both diagnostic and treatment purposes. Accordingly, there is evidence to suggest that EEG coherence may be an ideal target for neurotherapeutic interventions. For example, Thatcher et al. (2003) have suggested that coherence is a better predictor of IQ (and other neurocognitive constructs) than other EEG metrics including absolute power. Additionally, several studies have examined this question in children and adults with neurodevelopmental disorders. For example, Coben, Wright, Decker, and Morgan (2015) demonstrated coherence training improved reading performance above and beyond that of traditional school-based reading interventions. Coben (2008, as cited in Linden, \& Gunkelman, 2013) also demonstrated the efficacy of coherence training a sample of individuals with autism spectrum disorders. Furthermore, the authors completed a randomized control study of neurofeedback treatment for college students with ADHD, demonstrating significant changes in coherence, above and beyond that of other qEEG and behavioral metrics (Roberts \& Decker, 2015). Thus, not only does coherence provide a valid indicator of brain network connectivity that directly links to cognitive functioning, it may also be the best target for therapeutic outcomes.

\section{Clinical Applications for Coherence \\ in Assessment and Treatment}

\section{Theoretical}

Complex cognitive activity emerges from neuronal activity as part of an integrated network structure to exchange information throughout the brain (van den Heuvel \& Sporns, 2013). Although the degree to which disconnected brain networks manifest as discrepancies between different types of behavioral measures is not precisely known, reasonable inferences can be made based on the theory. Nonetheless, there are only a few cognitive theories that have formally integrated the role of brain connectivity with performance on cognitive measures. One exception is the Parieto-Frontal Integration Theory (P-FIT) of Intelligence (Jung \& Haier, 2007). This theory is derived from a review of literature on correlates of intelligence with a variety of brain imaging indicators, which provide a strong basis for intelligence being linked to the brain. Though current instantiations of the P-FIT model rely largely on MRI data, and thus do not take into account electrophysiological methods such as EEG and MEG that could shed light on the temporal dynamics of networks for intelligence, it provides a clear example of integrating connectivity with cognitive theory.

\section{Assessment and Identification}

Neuropsychological approaches to clinical disorders have recently grown in interest to practitioners (Decker, 2008). Historically, measures of cognition were limited to IQ scores. However, IQ scores were the basis of using such cognitive measures. Consistent with a contemporary neuropsychological view, specific cognitive deficits arise from connectivity problems in particular regions of the brain. The use of IQ scores, which is an amalgam of different cognitive tests compiled into a single score, lacks the specificity and sensitivity for capturing the exact cognitive deficits associated with different clinical disorders (Decker, Hale, \& Flanagan, 2013).

Supporting an NCC framework, causal links have been made between experimental changes in brain connectivity and behavior. For example, reading interventions, which enhanced brain connectivity in the left occipital-temporal region of the brain resulted in improved reading scores in children (Shaywitz \& Shaywitz, 2008). Similarly, meta-analytic research has supported clear changes in brain activity as a result of reading interventions (Barquero, Davis, \& Cutting, 2014). Specifically, researchers found that children with reading difficulties exhibited different amounts of functional connectivity in the frontal lobe 
compared to children without reading difficulties. However, after participating in different reading interventions, a difference in frontal connectivity no longer existed. Futhermore, recent literature suggests children with double-deficits (phonological and rapid naming deficits) have more atypical brain connectivity than children with only a single deficit (Norton et al., 2014), which demonstrates an additive relationship between atypical connectivity and learning problems. These findings indicate treatment outcomes of children with dyslexia, in comparison to a control group, are dependent on the normalization of brain connectivity in specific regions of the brain (Richards \& Berninger, 2008)-a concept that has major implications for directing future neurological interventions, such as coherence-based neurofeedback.

Within the NCC framework, an uneven profile of cognitive skills may correspond to deficits in network hub functionality in the brain. Some preliminary research may already suggest this is likely the case (Adelstein et al., 2011; Bassett \& Bullmore, 2006; Cole, Yarkoni, Repovš, Anticevic, \& Braver, 2012; Shimono, Mano, \& Niki, 2012; van den Huevel, Mandl, Luigjes, \& Hulshoff Pol, 2008; Zalesky \& Fornito, 2009).

Just as brain networks provide a common denominator for cognitive and academic weaknesses in phonology that change with intervention (Shaywitz \& Shaywitz, 2008), there is promise that other interventions involving different brain networks will be similarly effective. Within the NCC framework described here, the value of cognitive tests in assessing brain networks can be clarified. First, and historically noted, cognitive tests provide a behavioral indicator of the functional integrity of brain networks. Second, different cognitive tests provide an indicator of the integrity of different brain networks. Finally, coherence provides a more direct measure of the functional integrity of different brain networks. Unfortunately, no cognitive measure is pure and variance in performance can be attributed to contextual, socioeconomic, and educational sources. Coherence provides a more direct measure of brain connectivity to verify the neurophysiological basis of cognitive problems. However, the practical utility of both measures is in their correspondence with each other.

Ultimately all cognition is dependent on brain connectivity, and cognitive measures provide ecological validity for better understanding challenges someone might face in everyday life.
However, cognitive deficits as indicated by behavioral measures are ambiguous as to underlying causes. Coherence measures in conjunction with cognitive measures not only provide validation of diagnostic problems but also provide information to guide and select more targeted interventions. Evaluating the correspondence between cognitive test performance and brain hub involvement will likely be a future direction of neurocognitive and translational research.

In emphasizing the role of brain connectivity, the NCC perspective may provide an important theoretical foundation for guiding interventions. Essentially, children with SLD have weak connections in particular areas of the brain that reduce integration of associative learning that is involved in specific academic tasks. Similar functional deficits have been documented in a number of other neurodevelopmental and neurological disorders, as described above. Thus, interventions that facilitate connectivity of these brain regions should result in improved performance, or create the conditions for improving the efficiency of learning (as well as of attention, social skills attainment, etc., in other clinical disorders).

An important role emerging from NCC involves a revised understanding of attention, which has historically been difficult to define. Attention has been classically defined as a description of information held in awareness at a particular moment in time; it can become more or less focused and can shift; it involves both exogenous (environmental) influences as well as endogenous (within the person) influences. More contemporary research has found links between attention and brain connectivity. Specifically, attention is a cognitive mechanism that facilitates the binding or connectivity of different brain networks (Gootjes et al., 2006). Attention is important because it is influenced by both task demands and volitional control; thus, it is amendable to intervention.

Lastly, the NCC framework may provide a conceptual framework for explaining novel therapies that have been used in SLD and other clinical conditions. Neurofeedback (NF) has been one method used to directly change brain connectivity. This treatment involves a brain-computer interface for operant conditioning of brain activity, where patients are trained to direct their own EEG activity. Positive outcomes have been reported for various disabilities, including SLD. For instance, cases and experimental studies have demonstrated changes in brain connectivity from NF in children with dyslexia 
that resulted in improved spelling (Breteler, Arns, Peters, Giepmans, \& Verhoeven, 2010), improved reading (from 1.2 grade levels to upwards of 2 grade levels; Coben et al., 2015; Walker \& Norman, 2006), and improved phonological skills (Nazari, Mosanezhad, Hashemi, \& Jahan, 2012).

\section{Summary}

EEG Coherence is a metric derived from the electrical potentials in the brain to gauge the interrelationship between two electrodes. The measured relationship can be used to infer the degree of connectivity between two distant regions of the brain. As discussed throughout this manuscript, neuroimaging techniques, and EEG in particular, have become integral physiological metrics in the identification and study of various brain networks. More and more, research is focusing less on structural differences, in lieu of understanding how these interconnected structures communicate in order to process the increasingly complex environments that we encounter on a daily basis.

The integration of cognitive and neuroscience investigations of SLD is converging to suggest specific brain regions, or networks, are explicitly engaged cognitive tasks. Additionally, education requires integrated, or connected, brain networks dedicated to the differential processing demands in learning. The Neurocognitive Connectivity (NCC) framework is offered to synthesize the emerging theme of neuroscientific investigations. The NCC framework is demonstrated using examples of specific learning disabilities which involve problems in learning due to specific cognitive deficits. Furthermore, research is emerging to suggest children with SLD have specific atypical patterns of brain connectivity and these patterns of reduced connectivity in brain networks are the underlying cause of SLD. These atypical patterns of connectivity correspond to different displays of learning disabilities. Implications of viewing SLD as a brain network connectivity problem are discussed with relevance to theory, assessment, and intervention. While research supporting a disconnectivity model of SLD has been reinforced by neuroscientific investigations, there is also emerging evidence for the role of coherence metrics to detect atypical patterns of connectivity in brain networks for a broad array of neuropsychological and neuropsychiatric conditions. However, additional research in the applied and practical applications of the NCC model is necessary. While coherence can be derived from a variety of brain imaging methodologies, EEG and QEEG have numerous advantages within clinical applications. In addition to the general benefits over other neuroimaging methodologies related to temporal resolution, EEG and QEEG metrics are easy to obtain, inexpensive, noninvasive, cost-effective, and provide reliable and valid indicators of brain connectivity. Furthermore, coherence measures are ideal therapeutic targets for gauging treatment outcomes as well as the target of treatments involving neurofeedback.

Although continued research is needed to further investigate the ever-growing web of connectivity within the human brain, EEG coherence is a metric particularly well suited to this endeavor. Future research will likely continue to refine methodological aspects of coherence measures in identifying the best approaches to identify discrete brain networks within source space based on sensor level recordings. Additionally, coherence measures will likely factor into the formation of future models of cognition and provide a substantial role in not only shaping theoretical models of cognition but also therapeutic applications for individuals with cognitive deficits.

\section{References}

Adelstein, J. S., Shehzad, Z., Mennes, M., DeYoung, C. G., Zuo, X.-N., Kelly, C., ... Milham, M. P. (2011). Personality is reflected in the brain's intrinsic functional architecture. PLoS One, 6(11), e27633. http://dx.doi.org/10.1371 /journal.pone.0027633

Ashkenazi, S., Black, J. M., Abrams, D. A., Hoeft, F., \& Menon, V. (2013). Neurobiological underpinnings of math and reading learning disabilities. Journal of Learning Disabilities, 46(6), 549-569. https://dx.doi.org/10.1177/0022219413483174

Barquero, L. A., Davis, N., \& Cutting, L. E. (2014). Neuroimaging of reading intervention: A systematic review and activation likelihood estimate meta-analysis. PLoS One, 9(1), e83668. http://dx.doi.org/10.1371/journal.pone.0083668

Bassett, D. S., \& Bullmore, E. (2006). Small-world brain networks. The Neuroscientist, 12(6), 512-523. https://dx.doi.org/10.1177/1073858406293182

Berninger, V. W., Richards, T. L., \& Abbott, R. D. (2015). Differential diagnosis of dysgraphia, dyslexia, and OWL LD: Behavioral and neuroimaging evidence. Reading and Writing, 28(8), 1119-1153. http://dx.doi.org/10.1007/s11145-0159565-0

Bieńkiewicz, M. M. N., Brandi, M.-L., Goldenberg, G., Hughes, C. M. L., \& Hermsdörfer, J. (2014). The tool in the brain: Apraxia in ADL. Behavioral and neurological correlates of apraxia in daily living. Frontiers in Psychology, 5, 353. https://doi.org/10.3389/fpsyg.2014.00353

Boukrina, O., Barrett, A. M., Alexander, E. J., Yao, B., \& Graves, W. W. (2015). Neurally dissociable cognitive components of reading deficits in subacute stroke. Frontiers in Human Neuroscience, $\quad 9, \quad 298 . \quad$ https://dx.doi.org/10.3389 /fnhum.2015.00298

Bowyer, S. M. (2016). Coherence a measure of the brain networks: Past and present. Neuropsychiatric Electrophysiology, 2(1), 1. https://dx.doi.org/10.1186/s40810015-0015-7

Breier, J. I., Simos, P. G., Zouridakis, G., \& Papanicolaou, A. C. (1999). Temporal course of regional brain activation 
associated with phonological decoding. Journal of Clinical and Experimental Neuropsychology, 21(4), 465-476. https://dx.doi.org/10.5555/jcen.21.4.465.883

Breteler, M. H. M., Arns, M., Peters, S., Giepmans, I., \& Verhoeven, L. (2010). Improvements in spelling after qEEGbased neurofeedback in dyslexia: A randomized controlled treatment study. Applied Psychophysiology and Biofeedback, 35(1), 5-11. http://dx.doi.org/10.1007/s10484-009-9105-2

Bullmore, E., \& Sporns, O. (2009). Complex brain networks: Graph theoretical analysis of structural and functional systems. Nature Reviews Neuroscience, 10(3), 186-198. https://dx.doi.org/10.1038/nrn2618

Butterworth, B., Varma, S., \& Laurillard, D. (2011). Dyscalculia: From brain to education. Science, 332(6033), 1049-1053. https://dx.doi.org/10.1126/science.1201536

Cannon, R. L., Baldwin, D. R., Shaw, T. L., Diloreto, D. J., Phillips, S. M., Scruggs, A. M., \& Riehl, T. C. (2012). Reliability of quantitative EEG (qEEG) measures and LORETA current source density at 30 days. Neuroscience Letters, $\quad 518(1), \quad 27-31 . \quad$ https://dx.doi.org/10.1016 /j.neulet.2012.04.035

Cao, F., Bitan, T., \& Booth, J. R. (2008). Effective brain connectivity in children with reading difficulties during phonological processing. Brain and Language, 107(2), 91101. https://dx.doi.org/10.1016/j.bandl.2007.12.009

Chabot, R. J., Merkin, H, Wood, L. M., Davenport, T. L., \& Serfontein, G. (1996). Sensitivity and specificity of QEEG in children with attention deficit or specific developmental learning disorders. Clinical EEG and Neuroscience, 27(1), 26-34. https://dx.doi.org/10.1177/155005949602700105

Coben, R., Clarke, A. R., Hudspeth, W., \& Barry, R. J. (2008). EEG power and coherence in autistic spectrum disorder. Clinical Neurophysiology, 119(5), 1002-1009. https://dx.doi.org/10.1016/j.clinph.2008.01.013

Coben, R., Wright, E. K., Decker, S. L., \& Morgan, T. (2015). The Impact of Coherence Neurofeedback on Reading Delays in Learning Disabled Children: A Randomized Controlled Study. NeuroRegulation, 2(4), 168-178. https://dx.doi.org /10.15540/nr.2.4.168

Cole, M. W., Yarkoni, T., Repovš, G., Anticevic, A., \& Braver, T. S. (2012). Global connectivity of prefrontal cortex predicts cognitive control and intelligence. The Journal of Neuroscience, 32(26), 8988-8999. http://dx.doi.org/10.1523 IJNEUROSCI.0536-12.2012

Corsi-Cabrera, M., Galindo-Vilchis, L., del-Río-Portilla, Y., Arce, C., \& Ramos-Loyo, J. (2007). Within-subject reliability and inter-session stability of EEG power and coherent activity in women evaluated monthly over nine months. Clinical Neurophysiology, 118(1), 9-21. https://dx.doi.org/10.1016 /j.clinph.2006.08.013

Corsi-Cabrera, M., Solís-Ortiz, S., \& Guevara, M. A. (1997). Stability of EEG inter-and intrahemispheric correlation in women. Electroencephalography and Clinical Neurophysiology, 102(3), 248-255. https://dx.doi.org/10.1016 /s0013-4694(96)95179-6

Decker, S. L. (2008). School neuropsychology consultation in neurodevelopmental disorders. Psychology in the Schools, 45(9), 799-811. https://dx.doi.org/10.1002/pits.20327

Decker, S. L., Hale, J. B., \& Flanagan, D. P. (2013). Professional practice issues in the assessment of cognitive functioning for educational applications. Psychology in the Schools, 50(3), 300-313. https://dx.doi.org/10.1002/pits.21675

Dronkers, N. F. (1996). A new brain region for coordinating speech articulation. Nature, 184(6605), 159-161. http://dx.doi.org/10.1038/384159a0

Feldman, H. M., Lee, E. S., Yeatman, J. D., \& Yeom, K. W. (2012). Language and reading skills in school-aged children and adolescents born preterm are associated with white matter properties on diffusion tensor imaging.
Neuropsychologia, 50(14), 3348-3362. https://dx.doi.org /10.1016/j.neuropsychologia.2012.10.014

Fernández, T., Harmony, T., Rodríguez, M., Reyes, A., Marosi, E., \& Bernal, J. (1993). Test-retest reliability of EEG spectral parameters during cognitive tasks: I absolute and relative power. International Journal of Neuroscience, 68(3-4), 255261. https://dx.doi.org/10.3109/00207459308994280

Fields, R. D. (2008). White matter in learning, cognition and psychiatric disorders. Trends in Neurosciences, 31(7), 361370. https://dx.doi.org/10.1016/j.tins.2008.04.001

Fox, M. D., Snyder, A. Z., Vincent, J. L., Corbetta, M., Van Essen, D. C., \& Raichle, M. E. (2005). The human brain is intrinsically organized into dynamic, anticorrelated functional networks. Proceedings of the National Academy of Sciences of the United States of America, 102(27), 9673-9678. https://dx.doi.org/10.1073/pnas.0504136102

Friedrich, E. V. C., Suttie, N., Sivanathan, A., Lim, T., Louchart, S., \& Pineda, J. A. (2014). Brain-computer interface game applications for combined neurofeedback and biofeedback treatment for children on the autism spectrum. Frontiers in Neuroengineering, $\quad 7, \quad 21 . \quad$ https://dx.doi.org/10.3389 /fneng.2014.00021

Friston, K. J. (2011). Functional and effective connectivity: A review. Brain Connectivity, 1(1), 13-36. https://dx.doi.org /10.1089/brain.2011.0008

Geary, D. C. (2013). Early foundations for mathematics learning and their relations to learning disabilities. Current Directions in Psychological Science, 22(1), 23-27. http://dx.doi.org /10.1177/0963721412469398

Glasser, M. F., Smith, S. M., Marcus, D. S., Andersson, J. L. R., Auerbach, E. J., Behrens, T. E. J., ... Van Essen, D. C. (2016). The Human Connectome Project's neuroimaging approach. Nature Neuroscience. 19(9), 1175-1187. https://dx.doi.org/10.1038/nn.4361

Gootjes, L., Bouma, A., Van Strien, J. W., Scheltens, P., \& Stam, C. (2006). Attention modulates hemispheric differences in functional connectivity: Evidence from MEG recordings. Neurolmage, $\quad 30(1), \quad 245-253 . \quad$ http://dx.doi.org/10.1016 /j.neuroimage.2005.09.015

Grossmann, T. (2015). The development of social brain functions in infancy. Psychological Bulletin, 141(6), 1266-1287. http://dx.doi.org/10.1037/bul0000002

Gruzelier, J. H. (2014). EEG-neurofeedback for optimising performance. III: A review of methodological and theoretical considerations. Neuroscience \& Biobehavioral Reviews, 44, 159-182. http://dx.doi.org/10.1016/j.neubiorev.2014.03.015

Gudmundsson, S., Runarsson, T. P., Sigurdsson, S., Eiriksdottir, G., \& Johnsen, K. (2007). Reliability of quantitative EEG features. Clinical Neurophysiology, 118(10), 2162-2171. https://dx.doi.org/10.1016/j.clinph.2007.06.018

He, Q., Xue, G., Chen, C., Chen, C., Lu, Z.-L., \& Dong, Q. (2013). Decoding the neuroanatomical basis of reading ability: A multivoxel morphometric study. The Journal of Neuroscience, 33(31), 12835-12843. http://dx.doi.org/10.1523 IJNEUROSCI.0449-13.2013

Honey, C. J., Sporns, O., Cammoun, L., Gigandet, X., Thiran, J. P., Meuli, R., \& Hagmann, P. (2009). Predicting human resting-state functional connectivity from structural connectivity. Proceedings of the National Academy of Sciences, 106(6), 2035-2040. https://dx.doi.org/10.1073 /pnas.0811168106

John, E. R. (1977). Functional Neuroscience, Vol II: Neurometrics: Clinical Applications of Quantitative Electrophysiology. Hillsdale, NJ: Lawrence Erlbaum Associates.

Jung, R. E., \& Haier, R. J. (2007). The Parieto-Frontal Integration Theory (P-FIT) of intelligence: Converging neuroimaging evidence. Behavioral and Brain Sciences, 30(2), 135-154. https://dx.doi.org/10.1017/S0140525X07001185 
Kaiser, D. A. (2008). Functional Connectivity and Aging: Comodulation and Coherence Differences. Journal of Neurotherapy, 12(2-3), 123-139. http://dx.doi.org/10.1080 110874200802398790

Kanwisher, N., McDermott, J., \& Chun, M. M. (1997). The fusiform face area: A module in human extrastriate cortex specialized for face perception. The Journal of Neuroscience, 17(11), 4302-4311.

Keizer, A. W., Verment, R. S., \& Hommel, B. (2010). Enhancing cognitive control through neurofeedback: A role of gammaband activity in managing episodic retrieval. Neurolmage, 49(4), 3404-3413. $\quad$ http://dx.doi.org/10.1016 /j.neuroimage.2009.11.023

Koles, Z. J., Lind, J. C., \& Flor-Henry, P. (2010). Gender differences in brain functional organization during verbal and spatial cognitive challenges. Brain Topography, 23(2), 199204. http://dx.doi.org/10.1007/s10548-009-0119-0

Kucian, K., Ashkenazi, S. S., Hänggi, J., Rotzer, S., Jäncke, L., Martin, E., \& von Aster, M. (2014). Developmental dyscalculia: A dysconnection syndrome? Brain Structure and Function, 219(5), 1721-1733. https://dx.doi.org/10.1007 /s00429-013-0597-4

Kucian, K., \& von Aster, M. (2015). Developmental dyscalculia. European Journal of Pediatrics, 174(1), 1-13 https://dx.doi.org/10.1007/s00431-014-2455-7

Kucyi, A., Hove, M. J., Esterman, M., Hutchison, R. M., \& Valera, E. M. (2016). Dynamic Brain Network Correlates of Spontaneous Fluctuations in Attention. Cerebral Cortex, pii, bhw029. https://dx.doi.org/10.1093/cercor/bhw029

Lebel, C., Shaywitz, B., Holahan, J., Shaywitz, S., Marchione, K., \& Beaulieu, C. (2013). Diffusion tensor imaging correlates of reading ability in dysfluent and non-impaired readers. Brain and Language, 125(2), 215-222. https://dx.doi.org/10.1016 /j.bandl.2012.10.009

Linden, M., \& Gunkelman, J. (2013). QEEG-guided neurofeedback for autism: cClinical observations and outcomes. In M. F. Casanova, A. S. El-Baz, \& J. S. Suri (Eds.), Imaging the Brain in Autism (pp. 45-60). New York, NY: Springer.

Mišić, B., \& Sporns, O. (2016). From regions to connections and networks: New bridges between brain and behavior. Current Opinion in Neurobiology, 40, 1-7. https://dx.doi.org /10.1016/j.conb.2016.05.003

Miskovic, V., Schmidt, L. A., Boyle, M., \& Saigal, S. (2009). Regional electroencephalogram (EEG) spectral power and hemispheric coherence in young adults born at extremely low birth weight. Clinical Neurophysiology, 120(2), 231-238. http://dx.doi.org/10.1016/j.clinph.2008.11.004

Nazari, M. A., Mosanezhad, E., Hashemi, T., \& Jahan, A. (2012). The effectiveness of neurofeedback training on EEG coherence and neuropsychological functions in children with reading disability. Clinical EEG and Neuroscience, 43(4), 315-322. http://dx.doi.org/10.1177/1550059412451880

Norton, E. S., Black, J. M., Stanley, L. M., Tanaka, H., Gabrieli, J. D. E., Sawyer, C., \& Hoeft, F. (2014). Functional neuroanatomical evidence for the double-deficit hypothesis of developmental dyslexia. Neuropsychologia, 61, 235-246. https://dx.doi.org/10.1016/j.neuropsychologia.2014.06.015

Pascual-Marqui, R. D., Michel, C. M., \& Lehmann, D. (1994). Low resolution electromagnetic tomography: $A$ new method for localizing electrical activity in the brain. International Journal of Psychophysiology, 18(1), 49-65.

Pascual-Marqui, R. D., Lehmann, D., Koukkou, M., Kochi, K., Anderer, P., Saletu, B., ... Kinoshita, T. (2011). Assessing interactions in the brain with exact low-resolution electromagnetic tomography. Philosophical Transactions of the Royal Society A: Mathematical, Physical and Engineering Sciences, 369(1952), 3768-3784. https://dx.doi.org/10.1098 /rsta.2011.0081
Paulesu, E., Danelli, L., \& Berlingeri, M. (2014). Reading the dyslexic brain: Multiple dysfunctional routes revealed by a new meta-analysis of PET and fMRI activation studies. Frontiers in Human Neuroscience, 8, 830. https://dx.doi.org/10.3389/fnhum.2014.00830

Paulesu, E., Frith, U., Snowling, M., Gallagher, A., Morton, J., Frackowiak, R. S. J., \& Frith, C. D. (1996). Is developmental dyslexia a disconnection syndrome? Brain, 119(1), 143-157.

Pugh, K. R., Mencl, W. E., Shaywitz, B. A., Shaywitz, S. E., Fulbright, R. K., Constable, R. T., ... Gore, J. C. (2000). The angular gyrus in developmental dyslexia: Task-specific differences in functional connectivity within posterior cortex. Psychological Science, 11(1), 51-56. http://dx.doi.org /10.1111/1467-9280.00214

Qin, Y.-Y., Li, Y.-P., Zhang, S., Xiong, Y., Guo, L.-Y., Yang, S.-Q., ... Zhu, W.-Z. (2015). Frequency-specific Alterations of Largescale Functional Brain Networks in Patients with Alzheimer's Disease. Chinese Medical Journal, 128(5), 602-609. http://dx.doi.org/10.4103/0366-6999.151654

Quaglino, V., Bourdin, B., Czternasty, G., Vrignaud, P., Fall, S., Meyer, M. E., ... de Marco, G. (2008). Differences in effective connectivity between dyslexic children and normal readers during a pseudoword reading task: An fMRI study. Clinical Neurophysiology, 38(2), 73-82. http://dx.doi.org/10.1016 /j.neucli.2007.12.007

Richards, T. L., \& Berninger, V. W. (2008). Abnormal fMRI connectivity in children with dyslexia during a phoneme task: Before but not after treatment. Journal of Neurolinguistics, 21(4), 294-304. http://dx.doi.org/10.1016 /j.jneuroling.2007.07.002

Richardson, J. D., Fillmore, P., Rorden, C., LaPointe, L. L., \& Fridriksson, J. (2012). Re-establishing Broca's initial findings. Brain and Language, 123(2), 125-130. http://dx.doi.org/10.1016/j.bandl.2012.08.007

Rippon, G., Brock, J., Brown, C., \& Boucher, J. (2007). Disordered connectivity in the autistic brain: Challenges for the 'new psychophysiology'. International Journal of Psychophysiology, 63(2), 164-172. http://dx.doi.org/10.1016 /j.ijpsycho.2006.03.012

Roberts, A. M. \& Decker, S. L. (2015). Effects of Neurofeedback on Cognitive Profiles of College Students with $A D H D$. Plenary presentation at the 2015 of the International Society for Neurofeedback and Research (ISNR) $23^{\text {rd }}$ Conference, Denver, Colorado, USA.

Roberts, A. M., Fillmore, P., \& Decker, S. L. (2016). Clinical applicability of the test-retest reliability of qEEG coherence. NeuroRegulation, 3(1), 7-22, http://dx.doi.org /10.15540/nr.3.1.7

Rosenberg-Lee, M., Ashkenazi, S., Chen, T., Young, C. B., Geary, D. C. \& Menon, V. (2015). Brain hyper-connectivity and operation-specific deficits during arithmetic problem solving in children with developmental dyscalculia. Developmental Science, 18(3), 351-372. http://dx.doi.org /10.1111/desc. 12216

Shaw, J. C. (1984). Correlation and coherence analysis of the EEG: A selective tutorial review. International Journal of Psychophysiology, 1(3), 255-266. http://dx.doi.org/10.1016 10167-8760(84)90045-X

Shaywitz, B. A., Shaywitz, S. E., Pugh, K. R., Mencl, W. E., Fulbright, R. K., Skudlarski, P., ... Gore, J. C. (2002). Disruption of posterior brain systems for reading in children with developmental dyslexia. Biological Psychiatry, 52(2), 101-110.

Shaywitz, S. E., \& Shaywitz, B. A. (2008). Paying attention to reading: The neurobiology of reading and dyslexia. Development and Psychopathology, 20(4), 1329-1349. http://dx.doi.org/10.1017/S0954579408000631

Shaywitz, S. E., Shaywitz, B. A., Pugh, K. R., Fulbright, R. K., Constable, R. T., Mencl, W. E., ... Gore, J. C. (1998). Functional disruption in the organization of the brain for 
reading in dyslexia. Proceedings of the National Academy of Sciences of the United States of America, 95(5), 2636-2641.

Shimono, M., Mano, H., \& Niki, K. (2012). The brain structural hub of interhemispheric information integration for visual motion perception. Cerebral Cortex, 22(2), 337-344. http://dx.doi.org /10.1093/cercor/bhr108

Silani, G., Frith, U., Demonet, J.-F., Fazio, F., Perani, D., Price, C., ... Paulesu, E. (2005). Brain abnormalities underlying altered activation in dyslexia: A voxel based morphometry study. Brain, 128(10), 2453-2461. http://dx.doi.org/10.1093 /brain/awh579

Smith, S. M., Beckmann, C. F., Andersson, J., Auerbach, E. J., Bijsterbosch, J., Douaud, G., ... WU-Minn HCP Consortium. (2013). Resting-state fMRI in the Human Connectome Project. Neurolmage, 80, 144-168. http://dx.doi.org/10.1016 /j.neuroimage.2013.05.039

Sporns, O. (2011). The human connectome: A complex network. Annals of the New York Academy of Sciences, 1224, 109125. http://dx.doi.org/10.1111/j.1749-6632.2010.05888.x

Stam, C. J. (2014). Modern network science of neurological disorders. Nature Reviews Neuroscience, 15(10), 683-695. http://dx.doi.org/10.1038/nrn3801

Su, T.-W., Hsu, T.-W., Lin, Y.-C., \& Lin, C.-P. (2015). Schizophrenia symptoms and brain network efficiency: A resting-state fMRI study. Psychiatry Research: Neuroimaging, 234(2), 208-218. http://dx.doi.org/10.1016 /j.pscychresns.2015.09.013

Temple, E. (2002). Brain mechanisms in normal and dyslexic readers. Current Opinion in Neurobiology, 12(2), 178-183.

Thatcher, R. W. (2010). Validity and reliability of quantitative electroencephalography (qEEG). Journal of Neurotherapy, 14(2), 122-152. http://dx.doi.org/10.1080 $/ 10874201003773500$

Thatcher, R. W., Krause, P. J., \& Hrybyk, M. (1986). Corticocortical associations and EEG coherence: A twocompartmental model. Clinical Neurophysiology, 64(2), 123143.

Thatcher, R. W., Walker, R. A., Biver, C. J., North, D. N., \& Curtin, R. (2003). Quantitative EEG normative databases: Validation and clinical correlation. Journal of Neurotherapy, 7(3-4), 87121.

Thierry, G., Boulanouar, K., Kherif, F., Ranjeva, J. P., \& Démonet, J. F. (1999). Temporal sorting of neural components underlying phonological processing. Neuroreport, 10(12), 2599-2603.
Tucker, D. M., Roth, D. L., \& Bair, T. B. (1986). Functional Connections Among Cortical Regions: Topography of EEG Coherence. Electroencephalography and Clinical Neurophysiology, 63(3), 242-250. http://dx.doi.org/10.1016 10013-4694(86)90092-1

van den Heuvel, M., Mandl, R., Luigjes, J., \& Hulshoff Pol, H. (2008). Microstructural organization of the cingulum tract and the level of default mode functional connectivity. The Journal of Neuroscience, 28(43), 10844-10851. http://dx.doi.org/10.1523/JNEUROSCI.2964-08.2008

van den Heuvel, M. P., \& Sporns, O., (2013). Network hubs in the human brain. Trends in Cognitive Science, 17(12), 683-696. http://dx.doi.org/10.1016/j.tics.2013.09.012

Vandermosten, M., Boets, B., Poelmans, H., Sunaert, S. Wouters, J., \& Ghesquière, P. (2012). A tractography study in dyslexia: nNeuroanatomic correlates of orthographic, phonological and speech processing. Brain, 135(3), 935-948. http://dx.doi.org/10.1093/brain/awr363

Voytek, B., \& Knight, R. T. (2015). Dynamic network communication as a unifying neural basis for cognition, development, aging, and disease. Biological Psychiatry, 77(12), 1089-1097. http://dx.doi.org/10.1016 /j.biopsych.2015.04.016

Walker, J. E., \& Norman, C. A. (2006). The neurophysiology of dyslexia: A selective review with implications for neurofeedback remediation and results of treatment in twelve consecutive patients. Journal of Neurotherapy, 10(1), 45-55. http://dx.doi.org/10.1300/J184v10n01_04

Widjaja, E., Zamyadi, M., Raybaud, C., Snead, O. C., Doesburg, S. M., \& Smith, M. L. (2015). Disrupted Global and Regional Structural Networks and Subnetworks in Children with Localization-Related Epilepsy. AJNR American Journal of Neuroradiology, 36(7), 1362-1368. http://dx.doi.org/10.3174 lajnr.A4265

Zalesky, A., \& Fornito, A. (2009). A DTI-derived measure of cortico-cortical connectivity. IEEE Transactions on Medical Imaging, 28(7), 1023-1036. http://dx.doi.org/10.1109 /TMI.2008.2012113

Received: October 13, 2016

Accepted: October 22, 2016

Published: March 15, 2017 